\title{
Disrupted Rhythms of Life, Work and Entertainment Behaviours and Their Associations with Mental Health Problems under the Stress of COVID-19 Epidemic: A Survey in 5854 Chinese People with Different Backgrounds
}

Min Yang

Army Medical University

\section{Ping He}

Yongchuan Hospital of Chongqing Medical University

Xiaoming $\mathrm{Xu}$

the First Affiliated Hospital of Chongqing Medical University

Jing Wang

Army Medical University

Yanjun Wang

Army Medical University

Kaijun Liu

Army Medical University

Liangzhi Wen

Army Medical University

Xiao Xiao

Army Medical University

Yuqing $\mathrm{He}$

Army Medical University

Wen Tang

Army Medical University

Rong Zhao

Army Medical University

Yan Cao

Army Medical University

Linlin Chen

Army Medical University

Yanling Wei

Army Medical University 


\section{Chunhui Lan}

Army Medical University

\section{Xingwei Wang}

Army Medical University

\section{Bin Wang}

Army Medical University

\section{Wo Wang}

University-Town Hospital of Chongqing Medical University

\section{Mingming Zhao}

Three Gorges Hospital of Chongqing University

\section{Mei Zhao}

CAS Key Laboratory of Mental Health, Institute of Psychology

\section{Hui Lin}

Army Medical University

\section{Ruihua Yue}

Chongqing Emergency Medical Center

\section{Mingming Deng}

The Affiliated Hospital of Southwest Medical University

\section{Tianwei Deng ( $\nabla$ dengtw1986@163.com)}

Three Gorges Hospital of Chongqing University

\section{Li Kuang ( $\sim$ kuangli0308@163.com )}

the First Affiliated Hospital of Chongqing Medical University

\section{Dongfeng Chen ( $\square$ chendf1981@126.com )}

Army Medical University

\section{Research Article}

Keywords: Coronavirus disease 2019 (COVID-19), Rhythm disruption, Anxiety and Depression, Mental health problems, behaviours, Chinese people

Posted Date: May 9th, 2020

DOI: https://doi.org/10.21203/rs.3.rs-27400/v1

License: (c) (1) This work is licensed under a Creative Commons Attribution 4.0 International License. Read Full License 


\section{Abstract}

BACKGROUND and OBJECTIVE The rhythms of life, work and entertainment behaviours are considered as the external behavioural manifestations of biological rhythm. To evaluate the distinctive disrupted rhythms of behaviours and their associations with mental health problems in people with different backgrounds under the stress of COVID-19 epidemic.

SUBJECTS AND METHODS A cross-sectional study was conducted from 10-17 March 2020 under the stress of COVID-19 epidemic.A structured e-questionnaire containing general information,rhythm scale(subscale1 for life-work rhythms and subscale2 for entertainment rhythm) and Zung's self-rating depression and anxiety scale(SDS and SAS) were filled and the data were analysed.

RESULTS Overall 5854 participants were included.Significant differences were found in rhythm, SDS and SAS scores among people with different backgrounds (all $P<0.05$ ). Subjects with female gender and poor health status were mostly suffered from disrupted rhythms of life- work-entertainment behaviours, combined with depression and anxiety. Nurses and subjects being divorced or with chronic disease with psychosomatic diseases were mostly suffered from disrupted rhythms of life-work behaviours, combined with depression and anxiety. Subjects with aged 26-30 years, or annual income of 50,000-100,000CY were mostly suffered from disrupted rhythms of life-work combined with depression. Subjects with income over $300,000 \mathrm{CY}$ were mostly suffered from disrupted rhythm of entertainment combined with anxiety.The prevalence rates of depression and anxiety in people with the high-scores of rhythm disruption increased by $34.50 \%$ and $47.16 \%$, respectively, compared with those with low-scores.People with the high-scores of rhythm disruption had higher SDS and SAS scores, compared to those with low scores (all $P<0.001$ ). The independent related factors of disrupted rhythms included gender,age,marital status, health status,annual income and chronic diseases with psychosomatic diseases using logistic regression. The disrupted rhythms of life and work behaviours was positively correlated with both SDS and SAS scores.

CONCLUSIONS The disrupted rhythms of life, work and entertainment behaviours were closely associated with mental health problems. The disrupted rhythms of behaviours are frequent and fluxible,triggering more severe mental health problems under the stress of COVID-19 epidemic. The physicians should be aware of their importance when evaluating their interviewees or patients' mental health and achieving maximization of therapeutic efficacy by integrating the intervention of circadian rhythm and its behaviour.

\section{Introduction}

Coronavirus disease 2019 (COVID-19) is an acute respiratory infectious disease caused by a novel coronavirus1-3. Due to the strong infectivity of the coronavirus lack of effective treatments and vaccine, COVID-19 has spread rapidly and become a pandemic worldwide with serious consequences. Recent studies showed that COVID-19 affected people's psychological and metal status during this period of 
time 4-5. The biological rhythms, like an invisible body master "clock", are the recurrent rhythms existing in the living things, including the human beings.6-7 It has been demonstrated that the disruption of biological rhythms are closely related to mental health problemss, metabolic diseases, wound repair and tumour development, as well as the effects and toxicity of drugs. 8-13 It is well known that the rhythms of life, work and entertainment are considered as the external manifestations of biological rhythms.10,13-17 Despite preliminary reports on the effects of COVIND-19 on psychological and mental status4- 5 , there has been no report on rhythms variation and their associations with mental health problems. Therefore, the aim of the present study was to assess the rhythm disruption of life, work and entertainment activities and their associations with mental health problems in Chines people under stress of the epidemic of COVID-19, hoping to provide new ideas for the simultaneous two-way intervention from the perspective of biological rhythms and psychology. Additionally, the present study would lay an epidemiological foundation for the integration of biological rhythms with the diagnosis and treatment of psychosomatic diseases and the maximization of therapeutic efficacy.

\section{Methods}

\section{Participants and data collection}

An e-questionnaire was designed to investigate the rhythm activities and mental health problems in Chinese people with different backgrounds during the epidemic of COVID-19 through EQxiu online questionnaire platform. The survey was conducted from 10-17 March 2020 in twenty two provinces and five autonomous regions, four municipalities and two special administrative regions (i.e. Hong Kong and Macao) of China.All protocols are carried out in accordance with relevant guidelines and regulations and was approved by the Ethics Committee of the Army Medical Center (Approval No. 2019-144).During the survey, the participants were asked to read and check the informed consent and then continued to complete the questionnaire; those under 18 years old were asked to have the informed consent checked by their guardians, and then completed questionnaire. All completed questionnaires were received and checked for validity and completeness.

\section{Search strategy and selection criteria}

This survey was implemented by using a structured questionnaire that included three domains as described below.

General information Data on gender, age, marital status, educational level, annual pre-tax income, health status, current occupation, and chronic diseases were collected.

Rhythm scale The rhythm scale for life, work and entertainment activities , compiled by Margraf et al.,14 has been proved to have good reliability and validity in China.16,17 The scale consisting of 17 items was used to investigate the rhythm of individuals in six aspects: sleeping, getting up, mealtime, meeting guests, leisure activities and physical exercise on weekdays and weekends. The score was calculated by assigning scores of $1,2,3,4,5$ and 6 , in response to categories of very regularly, quite regularly, 
somewhat regularly, somewhat irregularly, quite irregularly, and very irregularly. The first eight items constituted subscale 1 which addressed issues on life and work rhythm, and was scored as the sum of the eight items. The last nine items with three problem groups constituted subscale 2 , which addressed issues on entertainment rhythm, and was scored as the sum of 6 questions. The lower the score was, the more regular the rhythm was.

Mental health problems Zung's self-rating anxiety scale (SAS) and self-rating depression scale (SDS) were used to evaluate the mental health problems of each participant.18 There were 20 items in SAS and SDS, and each item was graded to 1-4 reflecting never, often, sometimes, and always. The rough score was the sum of 20 questions, which was multiplied by 1.25 and rounded to get the standard score. The cut-off value was 50 for both SAS and SDS. The higher the score, the more obvious the anxiety or depression was; scores of 50-59, 60-69, and 70 points or more indicated mild, moderate and severe anxiety or depressive symptom, respectively.

\section{Statistical Analysis}

Data were analysed using the SPSS version 23.0 (SPSS, Chicago, IL, USA). Kolmogorov- Smirnov test was used to test the normal distribution of measurement data (scale score). The data of normal distribution was expressed by mean \pm standard deviation. $t$-test or one-way analysis of variance was used for comparison between groups and LSD method was used to compare the homogeneity of variance, Tamhane T2 test was used to compare the heterogeneity of variance, and pairwise comparison was made. The median (rang, minimum-maximum) or median (interquartile range, IQR) was used to represent the non-normal distribution data, and the nonparametric test (i.e. Mann-Whitney $\mathrm{U}$ test or Kruskal-Wallis test) was used for the comparison between two groups or more groups; the count data such as negative psychological detection were expressed by the number of cases and constituent ratio (\%). The $\chi 2$-test was used for the comparison between groups, and the Bonferroni correction method was used for pairwise comparison. Pearson correlation or Spearman correlation, where appropriate, was used to determine the correlation of anxiety or depression with rhythm scale scores. Linear regression analysis, after correcting the related factors, was used to determine the independent factors for the correlation of anxiety or depression with rhythm. A $P$ value of $<0.05$ was considered statistically significant.

\section{Results}

\section{Sociodemographic profile}

A total of 5872 people checked the e-questionnaire. Eighteen questionnaires with invalid and incomplete data were excluded, and thus data of 5854 participants who successfully completed questionnaires were further analysed.The general information on sociodemographic characteristics of the participants is listed in Table 1.

\section{Rhythms characteristic of life, work and entertainment}


The statistically significant differences were found among people with different gender, age, educational level, annual pre-tax income, health status, current occupation, and chronic diseases in subscale 1 and subscale 2 (all $P<0.05$ ) in each of the variables (Table 2). Specifically, the female gender and poor health status were most closely associated with disrupted rhythms of life, work and entertainment. The age group of 26-30 years, nurses and subjects with being divorced or widowed, the education level of senior high school, annual pre- tax income of 50,000-100,000 Chinese Yuan (CY), and chronic disease comorbid with psychosomatic diseases were mostly suffered from disrupted rhythms of life and work. The age group of older than 61 , sujects with education levels of master's degree or above, annual pre- tax income over 300,000 CY, and chronic disease without psychosomatic diseases were mostly suffered from disrupted rhythms of entertainment. Additionally, subscale1 scores were significantly higher in nurses than in doctors and in medical students than in non-medical students (both $P<0.05)$. (Table 2).

\section{Characteristics of anxiety and depression}

There were significant differences in anxiety and depression disorders among people with different genders, ages, marital status, educational levels, annual pre-tax incomes, health status, current occupation, and chronic diseases with or without psychosomatic diseases in each of the variables(all $P<$ 0.05) (Table 3).Specifically, nurses and subjects with female gender, being divorced or widowed, education levels of junior high school or below, poor health status, and chronic diseases comorbid with psychosomatic diseases were mostly suffered from depression and anxiety. Whereas the age group of 26-30 years, annual pre-tax income of 50,000-100,000 CY were mostly suffered from depression. The age group of 31-40 years and annual pre-tax income over 300,000 CY were mostly suffered from anxiety. Additionally, the SDS scores were significantly higher in medical students than in non-medical students and in farmers than in police (both $P<0.001$ ); There were significant differences(all $P<0.05$ ) in the prevalence of depression and anxiety, which were graded according to the SDS and SAS scores, among people with different genders, age groups, marital status, educational levels, annual pre-tax incomes, health status, current occupation, and chronic disease status (Tables 4A \& 4B).

\section{Correlation analysis between rhythm disruption and psychological disorders}

In subscale 1,27\% of the total number of samples were taken up from the lowest score as the low-score group (2390, $40.83 \%)$, and $27 \%$ of the total number of samples from the highest score were taken as the high-score group $(1574,26.89 \%)$. In subscale 2 , the low-score group with rhythm disruption of entertainment activities (1984,33.89\%), high-score group (1190,20.35\%). In Subscale1, the low-score group had significantly lower SDS and SAS scores, compared with high-score group (both $P<0.001$ ). Meanwhile, the prevalence of depression and anxiety between the high and low-score groups in subscale1 were significantly different ( $\chi 2=861.974$ and 584.577 , respectively, both $P<0.001)$. In subscale 2 , compared with the high- score group, the low-score group had significant lower SAS scores $(P=0.001)$. Moreover, the prevalence of depression was significantly different $(\chi 2=10.706, P=0.013)$ between the high and low-score groups in subscale2. Under the stress of COVID-19 epidemic, the prevalence rate of 
depression and anxiety in the high-score groups with rhythm disruption increased by $34.50 \%$ and $47.16 \%$, respectively, compared with the low-score group.

Eight factors including gender, age, marital status, education, annual income, health status, current occupation and chronic diseases were included in the linear regressive analysis. It was shown that six of these factors were independently associated with life and work rhythm disruption (all $P<0.001$ ); annual income, marital status and psychosomatic diseases were positively correlated with subscale1 score, while age, gender and health status were negatively correlated with subscale1 score. In addition, five factors were independently associated with entertainment rhythm disruption (all $P<0.05$ ); marital status and current occupation were negatively correlated with subscale 2 score, while gender, age and education were positively correlated with subscale 2 score.

The subscale1 score was positively correlated with the scores of SDS and SAS, and the correlation coefficients were 0.550 and 0.544 , respectively $(P<0.001$,Table 5), indicating that disrupted rhythms of life and work were associated with increased levels of depression and anxiety. There was a weak negative association between subscale2 score and SDS scores, with a correlation coefficient of -0.037 ( $P=$ 0.001 ,Table 5 ). When the subscale 1 or 2 scores were taken as dependent variables, the SDS and SAS scores were taken as independent variables, and age, gender, education and occupation status were taken as confounding factors, the association between the subscale 1 or 2 scores and SDS and SAS scores remained unchanged after correcting confounding factors in each model (all $P<0.05$ ) (Tables 6A $\& 6 \mathrm{~B})$.

\section{Discussion}

\section{Analysis of rhythm characteristics and its sociological value under the stress of COVID-19 epidemic}

Biological "clock" and its rhythms are a kind of prediction and early warning mechanism selected by the environment in the process of evolution over the years. Species which can grasp the law of environmental changes and foresee what happens have the advantages of survival and reproduction.11,19,20 A study published in Lancet Psychiatry showed that the early identification and intervention of rhythm disruption have important clinical significance.10 Previous studies have shown that rhythm disruption is associated with poor mental health problems, such as depression, anxiety, low happiness and strong loneliness.10,14-17

The present study found that the rhythm disruption of life, work and entertainment in females were worse than that in males. This gender difference may be attributed to the fact that oestrogen would affect the balance of hypothalamus-pituitary-adrenal axis (HPA axis) under COVID-19 epidemic stress, making females are more sensitive to HPA axis than males.21-22 Additionally, menopausal women's circadian and endocrine disorders are more likely to lead to rhythm disruption.

In this study, a significant difference was found among people with different ages in the rhythm scale. This age-related difference may be explained, First, it is known that people aged 26-40 years old are the 
main force of the society, and actively participate in the activities of various industries, which promotes the consumptions, cultural creativity and economic development. However, they had to stay at home or fight against the epidemic due to the sudden outbreak of COVID-19 epidemic.23-24. Second, people under 18 and those aged 18-25 years old participating in the present study were mainly students. During the COVID-19 epidemic, they participated regularly in home network courses and distance educations organized by education supervisors. 25 So, these groups of people remained their regular life, study rhythms during the epidemic. Third, the phenomenon that people aged 18-25 and 26-40 years old maintained their regular entertainment rhythm, such as home physical exercise, literary and artistic activities, advocated by the responsible cultural and sports executives. Fourth, it has been shown that people with poor health status or older than 61 years old have more basic diseases, weaker immune function, and low ability to fight pathogens or adapt to rhythm replacement.26-27 Thus, they did not dare to come out to participate in outdoor sports during the epidemic, and thus their the life, work and entertainment rhythms were all affected by the epidemic.

The present study found that the people with low educational levels or low annual income were most like to have irregular life and work rhythms. This may be explained by the assumption that these people have weak competitiveness in the job market and are currently waiting for the resumption of work and production. Moreover, they have not yet adapted to the new business models. Interestingly, the people with master's degree or above were most like to have irregular entertainment rhythm. This might be resulted from the fact that these intelligent people mainly concentrated on medical care, education, scientific research, artificial intelligence engineering and so on., and thus, they had little leisure time during the COVID-19 epidemic.28 In addition, The most irregular in the divorced or widowed group might be related to the disintegration of the family, loose family relations, economic problems, and the problem of raising children as a single parent.29 Especially, the isolation during COVID-19 epidemic may have aggravated the fear for the unknown future, loneliness, helplessness, or irregular eating and drinking.

The life and work rhythms of nurses were the most irregular among different occupations. The main reasons were assumed as follows. First was the particularity of nursing work.30-31 Nursing staff reserves are relatively insufficient and multiple shifts work became more frequently during the COVID-19 epidemic. Second, the risk and relative closure of the work environment, indoor equipment and noisy environment, and closeness to patients all increased the tension and anxiety.Third, nurses worked at high intensity and high pressure. Nurses needed to deal with patients' emergencies immediately, especially for critically ill patients who needed a ventilator, tracheotomy, or extracorporeal membrane oxygenation (ECMO). Nurses not only did therapeutic nursing, but also did some life care, disinfection and isolation.

\section{Analysis of psychological problems under the stress of COVID-19 epidemic.}

The present study found that there were significant differences in psychological disorders among in people with different backgrounds. First, the HPA axis was more responsive to stress in women than in men, as described earlier.21,22 Second, people with low education levels were not competitive in the work market, and thus more likely to be affected by the epidemic and face the grim employment situation. 
Moreover, they did not have sufficient judgement while facing various, overloaded and timeless information on COVID-19 epidemic, and easily felt depressive and anxious. Third, people aged 26 to 40 years, the backbone of the society and families, especially those fighting against the epidemic resulted in psychological impact. The depression and anxiety existed with a high prevalence in the group of medical staff, especially in nurses. The reasons might be the follows.30-31 First, COVID-19 is a new infectious disease with a sudden epidemic, and thus medical staff had no or little knowledge on its route of transmission, prevention and treatment, and thus worried about the infected risk. Second, medical staff were difficult to balance between work and family, and especially after working in the isolation ward, they had to be separated themselves from the family for a long time. Third, when the medical staff

encountered the new confirmed or suspected cases every day, witnessing patients in great pain but failing to save their lives despite their best efforts, they would feel frustrated and guilty. Fourth, the mental health disorders in nurses were the most serious among the various occupations, this may be related to their special occupation, working environment, high-risk and arduous tasks during COVID-19 epidemic, as mentioned earlier.

\section{Analysis of association between the disrupted rhythms and psychological problem}

A previous study observed an association of disrupted circadian rhythmicity with mood disorders by wearing a wrist-worn accelerometer for 7 days, and found that circadian disruption could increase the risk of depression, bipolar disorder; compared with people who worked normally, the prevalence rate of mood disorders was 6-10\% higher in people with circadian disorders. 10 However, the present study found that the prevalence rates of depression and anxiety in high-score group with rhythm disruption of life-work increased by $34.50 \%$, and $47.16 \%$, respectively, suggesting that the rhythm disruption under the stress of the COVID-19 epidemic had triggered a higher and more risk of mental health problems.

Our research further found that the scores of life and work rhythms were positively correlated with SDS and SAS scores. This association was theoretically based on the following aspects. First, the hypothesis of "social timing factor theory" holds that stress is a regulating factor of biological clock, which leads to various adverse mental health.32 Second, the "knockout monkey" model had circadian rhythm disruption with analogous schizophrenia symptom after knocking out the core rhythm gene of BMAL1 .33Third, it has been reported that the disrupted rhythm of suprachiasmatic nucleus leads to helplessness and despair, and increases depression and anxiety-related behaviourss.34 Fourth, the function of HPA axis is also regulated by the biological clock.35 Therefore, we speculate that if the treatment of depression and anxiety disorders is organically combined with the regulation of the biological clock to restore the biological rhythms, then the efficacy of antidepressants would be significantly improved.

In general, entertainments, such as regular physical and cultural activities could relieve adverse emotion such anxiety and depression.36-37 However, in this study, for the special nurse group with rhythms disruption of life and work, it seems difficult to improve anxiety and depression through regular entertainment under stress of COVID-19 epidemic. Thus, it is necessary to further evaluate rhythm disruption and mental health problems of the target population, identify the possible signs of group 
psychological crisis, and incorporate psychological crisis intervention with adjustment of disrupted rhythms of life, work and entertainment timely for the high-risk groups to avoid extreme events, such as self-injury or even suicide.

\section{Synchronized intervention strategy for rhythm disruption and psychological problems.}

Currently, extensive attention has been paid to the biological clock in the field of psychiatry and psychology due to its importance.6,10-12,38,39 We hope that future research on optimizing the functions of the biological clock can develop a new area of "rhythmic medicine". If the circadian rhythm could be more effectively integrated with the warning, diagnosis and treatment of psychosomatic diseases, then, mental health might be improved, psychosomatic diseases prevented, and the therapeutic efficacy maximized. Therefore, based on the findings of the present study, we recommend reversing the disruption of life, work and entertainment rhythms, cooperating with classical psychological therapy, and intervening the psychological impact caused by COVID-19 epidemic.

Presently, health-care workers are the most valuable resource of each country, their safety must be ensured. For medical groups, especially nurses with the rhythm disruption, depression and anxiety, we would recommend the following the interventions. First, the adjustment of the daily rhythm starts from the virtuous circle such as the habit of going to bed early and getting up early, exercising appropriately, changing your posture, basking in the sun and listening to the music. Second, drugs with adjusting the biological clock should be considered for sleep disorders caused by nurses' shift system, when necessary.40,41 Third, the rhythm of work should be changed properly such as adjusting the working time, and transferring work from the high stress job to the low stress job. Fourth, the eating patterns and food should be adjusted.Previous studies have shown that regular eating and various metabolites promote the periodic colonization of the intestinal bacteria on the mucosal surface, and then alleviate various adverse mental problems through the intestinal flora-gut-brain axis42,43. Fifth, as nurses had high vulnerability to COVID-19, adequate provision of personal protective equipment is necessary and hospital-specific protocols should be established to reduce the risk of nurses' infection in the interactions with patients.44-45

\section{Limitation}

There are a few limitations in the present study. First, all the subjects were from China, and thus the findings should be interpreted with caution for colleagues outside China. Further investigation with regional or even international cooperation is required to draw more representative conclusions. Second, the number of participants older than 61 was small in the present study, mainly due to inability to use the smart phone, worry about operational errors, poor eyesight and so on. In the future, we would like to design a special intelligent voice system in a survey for the elderly. 


\section{Conclusions}

This is the first study, from a new perspective of biological clock rhythm-social activity regularity, to investigate the life, work and entertainment rhythms in people with different backgrounds and their associations with mental health problems under the stress of COVID-19 epidemic. The findings would provide new ideas for the intervention of COVID-19 epidemic and any sudden, threatening or catastrophic life events that human beings maybe face in the future. Additionally, the findings of the present study laid an epidemiological foundation for the integration of the biological clock rhythm with the diagnosis and treatment of psychosomatic diseases and the maximization of therapeutic efficacy.

Under the stress of COVID-19 epidemic, there existed distinctive rhythm disruption among Chinese people with different backgrounds. The rhythm disruption was closely related to psychological problems, suggesting that the disruption of life and work rhythms can predict mental health status. For nurses, with mostly obvious rhythm disruption of life and work rhythms, it seems difficult to improve anxiety and depression through regular entertainment rhythm under the stress of COVID-19 epidemic. The cooperation of adjusting disrupted rhythm of life, work and entertainment, and psychological therapy could improve the clinical effectiveness of psychological crisis intervention.

\section{Declarations}

\section{Author Contributions}

Min Yang,Ping He,Xiaoming Xu and Jing Wang formulated the study design,carried out this study and wrote the manuscript.Yanjun Wang, Kaijun Liu, Liangzhi Wen, Xiao Xiao,Yuqing He and Wen Tang collected data. Rong Zhao, Yan Cao, Linlin Chen,Yanling Wei and Chunhui Lan assisted with data collection and literature.Xingwei Wang, Bin Wang and Wo Wang prepared the tables and format adjustment.Minmin Zhao, Mei Zhao, Hui Lin,Ruihua Yue and Ming-Ming Deng contributed to data analysis and data interpretation.Tianwei Deng,Li Kuang and Dongfeng Chen conceived the study and edited the manuscript.

Conflict of Interest Disclosures: None reported.

Funding/Support: This study was supported by the grant from Chongqing basic research and foreword exploration project ( Grant No.CSTC2018jcyjAX0620).

Role of the Funder/Sponsor: The sponsors had no role in the design and conduct of the study; collection, management, analysis, and interpretation of the data; preparation, review, or approval of the manuscript; and decision to submit the manuscript for publication.

Additional Contributions: Zhihua Gao, PhD and Jinxia Zhu, PhDprovided valuable assistance with on questionnaire design.Kai Guo provided the on-line technical support.We are grateful for Medjaden 
Bioscience Limited for its assistance in the preparation of the manuscript. None of these contributors were compensated for this work.

\section{References}

1. Zhou, et al. Clinical course and risk factors for mortality of adult inpatients with COVID-19 in Wuhan, China: a retrospective cohort study. Lancet 395,1054-1062 (2020)

2. Huang, et al. Clinical features of patients infected with 2019 novel coronavirus in Wuhan, China. Lancet (London, England) 395, 497-506 (2020)

3. Li,Q. et Early transmission dynamics in Wuhan, China, of novel coronavirus-infected pneumonia. $N$ Eng/ J Med 382,1199-207 (2020)

4. Yao,H. et Patients with mental health disorders in the COVID-19 epidemic. Lancet Psychiatry 7, e21 (2020)

5. Lai, et al. Factors associated with mental health outcomes among health care workers exposed to coronavirus disease 2019. JAMA Network Open 3, e203976 (2019).

6. Sehgal, Physiology Flies with Time. Cell 171,1232-1235(2017).

7. Huang, R. C. The discoveries of molecular mechanisms for the circadian rhythm: The 2017 Nobel Prize in Physiology or Medicine. Biomed J 41, 5-8 (2018).

8. Montaigne, et al. Daytime variation of perioperative myocardial injury in cardiac surgery and its prevention by Rev-Erbalpha antagonism: a single-centre propensity-matched cohort study and a randomised study. Lancet 391,59-69(2018).

9. Michael, A. K. et al. Cancer/Testis antigen PASD1 silences the circadian clock. Mol Cel/ 58,743754(2015).

10. Lyall, M. et al. Association of disrupted circadian rhythmicity with mood disorders, subjective wellbeing, and cognitive function: a cross-sectional study of 91105 participants from the UK Biobank. Lancet Psychiatry 5,507-514(2018).

11. Pilz, K. et al. Rhythmicity of mood symptoms in individuals at risk for psychiatric disorders. Sci Rep 8,11402(2018).

12. Palagini, et al. Association between affective temperaments and mood features in bipolar disorder II: The role of insomnia and chronobiological rhythms desynchronization. J Affect Disord 266,26372(2020).

13. Koritala, S.C. et al. The human circadian clock from health to economics. Psych J 2018; 7, 176-196.

14. Margraf, J. et al. Social rhythm and mental health: a cross-cultural comparison. PloS One 11,e0150312(2016). 
15. Velten, et al. Lifestyle choices and mental health: a representative population survey. BMC Psycho/ $\mathbf{2}$, 58(2014).

16. Velten, et al.Lifestyle choices and mental health: a longitudinal survey with German and Chinese students. BMC Public Health 18,632(2018).

17. Cai, D. et al. The bidirectional relationship between positive mental health and social rhythm in college students:a three-year longitudinal Front Psycho/ 8,1119(2017).

18. Dunstan, A. et al. Screening for anxiety and depression: reassessing the utility of the Zung scales. BMC Psychiatry 17,329(2017).

19. Patke, et al. Molecular mechanisms and physiological importance of circadian rhythms. Nat Rev Mol Cell Biol 21, 67-84(2020).

20. Vetter, Circadian disruption: What do we actually mean? Eur J Neurosci 51,531-550(2020).

21. Fuss, J. et al. Does sex hormone treatment reverse the sex-dependent stress regulation? A longitudinal study on hypothalamus-pituitary-adrenal (HPA) axis activity in transgender individuals. Psychoneuroendocrinology 104,228-237(2019).

22. Nishimura, et al. Effect of oestrogen-dependent vasopressin on HPA axis in the median eminence of female rats. Sci Rep 9, 5153(2019).

23. Zitting, K.M. et al. Young adults are more vulnerable to chronic sleep deficiency and recurrent circadian disruption than older adults. Sci Rep 8,11052(2018).

24. Zhao, K. et al. Negligible risk of the COVID-19 resurgence caused by work resuming in China (outside Hubei): a statistical probability J Public Health (Oxf) 2020.

25. Liu, et al.Response to the COVID-19 Epidemic: The Chinese Experience and Implications for Other Countries. International journal of environmental research and public health 17(2018).

26. Yan, et al. Association between anxiety, depression, and comorbid chronic diseases among cancer survivors. Psycho-oncology 28,1269-1277(2019).

27. Zhang, et al. Association of circadian rhythm of blood pressure and cerebral small vessel disease in community-based elderly population. J Gerontol A Biol Sci Med Sci 74,1322-1330(2019).

28. Krause, N. Assessing the relationships among stress, god-mediated control, and psychological distress/well-being: Does the level of education matter? J Soc Psychol 159,2-14(2019).

29. Auersperg, et al. Long-term effects of parental divorce on mental health - A meta-analysis. $J$ Psychiatr Res 119,107-115(2018).

30. Kang, L. et al.Impact on mental health and perceptions of psychological care among medical and nursing staff in Wuhan during the 2019 novel coronavirus disease outbreak: a cross-sectional study. Brain Behav Immun (2020).

31. Jensen, H. I. et al. The impact of shift work on intensive care nurses' lives outside work: A crosssectional J Clin Nurs 27, e703-e709(2018). 
32. Grandin, D. et al. The social zeitgeber theory, circadian rhythms, and mood disorders: review and evaluation. Clin Psychol Rev 26,679-694(2006).

33. Qiu, et al. BMAL1 knockout macaque monkeys display reduced sleep and psychiatric disorders. National Science Review 6, 87-100(2019)

34. Nagy, D. et al. Melatonin adjusts the expression pattern of clock genes in the suprachiasmatic nucleus and induces antidepressant-like effect in a mouse model of seasonal affective disorder. Chronobiol Int 32,447-457(2015).

35. Shaheen, et al. Victims of war-Psychoendocrine evidence for the impact of traumatic stress on psychological well-being of adolescents growing up during the Israeli-Palestinian conflict. Psychophysiology 57, e13271(2020).

36. Teo, et al. A feasible and acceptable multicultural psychosocial intervention targeting symptom management in the context of advanced breast cancer. Psychooncology 29,389-397(2020).

37. Bell, S. L. et al. The relationship between physical activity, mental wellbeing and symptoms of mental health disorder in adolescents: a cohort Int J Behav Nutr Phys Act 16,138(2019).

38. Angerer, et al Night work and the risk of depression. Dtsch Arztebl Int 114,404-411(2017).

39. Abbott, S. M. et al. Health implications of sleep and circadian rhythm research in 2017. Lancet Neurol 17,17-18(2018).

40. Leung, et al. Shift work, chronotype, and melatonin patterns among female hospital employees on day and night shifts. Cancer Epidemiol Biomarkers Prev 25, 830-838(2016).

41. Vetter, et al. Aligning work and circadian time in shift workers improves sleep and reduces circadian disruption. Curr Biol 25,907-911(2015)

42. Sanada, K. et al. Gut microbiota and major depressive disorder: A systematic review and metaanalysis. J Affect Disord 266,1-13(2020)

43. Thaiss, A. et al. Microbiota diurnal rhythmicity programs host transcriptome oscillations. Cell 167,1495-1510 e12(2016)

44. Huang, et al. Special attention to nurses' protection during the COVID-19 epidemic. Crit Care $\mathbf{2 4 , 1 2 0 ( 2 0 2 0 )}$

45. The Lancet. COVID-19: protecting health-care workers. Lancet 395,922(2020)

\section{Tables}

Due to technical limitations, Tables 1-6 are only available as a download in the supplemental files section

\section{Supplementary Files}

This is a list of supplementary files associated with this preprint. Click to download. 
- Tables.pdf

Page 15/15 